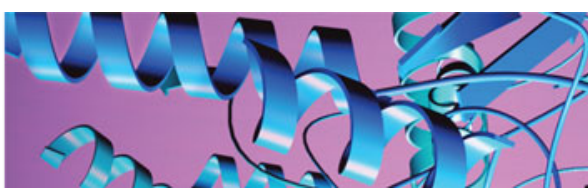

REPORT

\title{
Secondary nucleation of monomers on fibril surface dominates $\alpha$-synuclein aggregation and provides autocatalytic amyloid amplification
}

\author{
Ricardo Gaspar ${ }^{1,2}$, Georg Meist ${ }^{3}$, Alexander K. Buell ${ }^{3,4}$, Laurence Young ${ }^{5}$, Clemens F. Kaminski ${ }^{5}$, \\ Tuomas P. J. Knowles ${ }^{3}$, Emma Sparr ${ }^{1 *}$ and Sara Linse ${ }^{2 *}$ \\ ${ }^{1}$ Department of Physical-Chemistry, Lund University, Lund, Sweden \\ ${ }^{2}$ Department of Biochemistry and Structural Biology, Lund University, Lund, Sweden \\ ${ }^{3}$ Department of Chemistry, University of Cambridge, Cambridge, UK \\ ${ }^{4}$ Institute of Physical Biology, University of Düsseldorf, Düsseldorf, Germany \\ ${ }^{5}$ Department of Chemical Engineering and Biotechnology, University of Cambridge, Cambridge, UK \\ Quarterly Reviews of Biophysics (2017), 50, e6, page 1 of 12 doi:10.1017/S0033583516000172
}

Abstract. Parkinson's disease (PD) is characterized by proteinaceous aggregates named Lewy Bodies and Lewy Neurites containing $\alpha$-synuclein fibrils. The underlying aggregation mechanism of this protein is dominated by a secondary process at mildly acidic $\mathrm{pH}$, as in endosomes and other organelles. This effect manifests as a strong acceleration of the aggregation in the presence of seeds and a weak dependence of the aggregation rate on monomer concentration. The molecular mechanism underlying this process could be nucleation of monomers on fibril surfaces or fibril fragmentation. Here, we aim to distinguish between these mechanisms. The nature of the secondary processes was investigated using differential sedimentation analysis, trap and seed experiments, quartz crystal microbalance experiments and super-resolution microscopy. The results identify secondary nucleation of monomers on the fibril surface as the dominant secondary process leading to rapid generation of new aggregates, while no significant contribution from fragmentation was found. The newly generated oligomeric species quickly elongate to further serve as templates for secondary nucleation and this may have important implications in the spreading of PD.

\section{Introduction}

Protein misfolding and aberrant aggregation processes that elude cellular maintenance mechanisms can result in major disturbances of cellular processes. This may lead to protein aggregation diseases, for example Parkinson's disease $(\mathrm{PD})$, the prevalence of which is increasing (Dobson, 2003). In PD, the formation and deposition of amyloid fibrils by the protein $\alpha$-synuclein ( $\alpha$-syn) is the pathological hallmark associated with degeneration of dopaminergic neurons in the substantia nigra (Fink, 2006), and other brain regions. Neurodegeneration is believed to initiate at

* Authors for correspondence: Emma Sparr, Department of Physical-Chemistry, Lund University, Lund, Sweden and Sara Linse, Department of Biochemistry and Structural Biology, Lund University, Lund, Sweden. Email: emma.sparr@fkem1.lu.se and sara.linse@biochemistry.lu.se the synapse, and once started, the disease spreads without remission until reaching a terminal phase (Danzer et al. 2009; Schulz-Schaeffer, 2010). Cell death appears to result from the aggregation process per se or the presence of oligomeric aggregates that impair neurotransmission (Lesné et al. 2006; Shankar et al. 2008; Wakabayashi et al. 2007; Winner et al. 2011). Indeed, toxic forms may arise in a reaction involving both monomeric and fibrillar species (Jan et al. 2011), generating oligomers at the fibril surface (Cohen et al. 2013).

$\alpha$-Syn is a natively unfolded 140 amino acid protein that exists abundantly in neuronal cells, where it is located in the proximity of vesicles within the presynaptic terminals (Izawa et al. 2012). $\alpha$-Syn comprises $1 \%$ of total cytosolic 
protein in the nervous system (Stefanis, 2012) with an estimated intracellular concentration ranging from 30 to $60 \mu \mathrm{M}$ (Rabe et al. 2013). The physiological functions of $\alpha$-syn are still unclear, but are likely related to vesicle trafficking and release (Bisaglia et al. 2009). The onset of PD is also unclear, but has been suggested to be related to $\alpha$-syn levels above a critical aggregation concentration (Galvagnion et al. 2015; Pinotsi et al. 2016; Rabe et al. 2013). The amyloid fibrils formed from recombinant $\alpha$-syn in vitro are highly similar to those extracted from PD patients in terms of morphology and size (Conway et al. 1998). In vitro experiments have shown that the aggregation of $\alpha$-syn is very sensitive to solution conditions, such as temperature (Uversky et al. 2001), $\mathrm{pH}$ (Buell et al. 2014), salt concentration (Munishkina et al. 2004) and the presence of cofactors (Ashmad et al. 2012). In addition, the presence of surfaces, e.g. air-liquid interface for which $\alpha$-syn has high affinity, has been shown to influence aggregation kinetics in vitro (Campioni et al. 2014). The primary nucleation of $\alpha$-syn can be accelerated by the presence of lipid membranes (Galvagnion et al. 2015; Grey et al. 2015), surfactant micelles (Giehm \& Otzen, 2010) and nanoparticles (Vácha et al. 2014). Mechanical agitation enhances fibril fragmentation and increases the air-water interfacial area, which may promote heterogeneous primary nucleation (Campioni et al. 2013; Giehm \& Otzen, 2010). Quiescent conditions may therefore be preferred in mechanistic studies.

The formation of amyloid fibrils occurs through a nucleation-dependent polymerization reaction. Underlying the macroscopically observable sigmoidal growth curves are several possible microscopic steps occurring simultaneously: primary nucleation of monomers in solution or on surfaces, elongation of fibrils through the addition of (a) Fragmentation

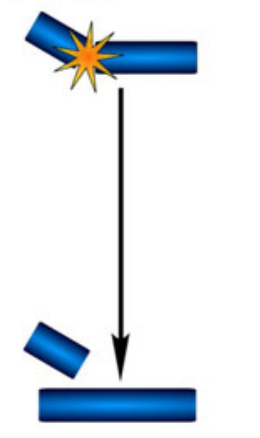

(b) Secondary Nucleation

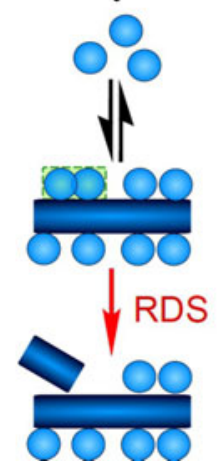

Fig. 1. Schematic depictions of the two possible secondary processes in the aggregation of $\alpha$-syn at mildly acidic $\mathrm{pH}$ conditions. (a) Fibrils multiply through fragmentation. (b) Fibrils catalyze the formation of new aggregates from monomer on their surface. In a saturated secondary nucleation reaction the initial monomerdependent (attachment) step is so fast that the second monomerindependent (rearrangement or detachment) step becomes the rate determining step (RDS). monomers to fibril ends, secondary nucleation of monomers on the surface of already existing fibrils and fibril breakage (Arosio et al. 2014; Pinotsi et al. 2014). In each case, detailed kinetic studies are needed to reveal the relative importance of these steps. For $\alpha$-syn, mechanistic studies under quiescent conditions are difficult because homogeneous primary nucleation in bulk solution is undetectably slow, a behavior very different from that observed for many other aggregating proteins, including $\mathrm{A} \beta 42$ (Arosio et al. 2015; Cohen et al. 2013), A $\beta 40$ (Meisl et al. 2014), actin (Oosawa \& Asakura, 1975) and some prions (Collins et al. 2004; Tanaka et al. 2006). Therefore, the initiation of in vitro aggregation requires the presence of seeds or other surfaces that enhance nucleation; here we have used pre-formed $\alpha$-syn fibrils.

\section{Outline of the problem}

The formation of amyloid fibrils by the intrinsically disordered protein $\alpha$-syn is a hallmark of PD. There is a correlation between spreading of amyloid and neuronal death. It is therefore of utmost importance to characterize the underlying microscopic steps of the assembly reaction that lead to the conversion of soluble $\alpha$-syn into its insoluble amyloid fibrils. At mildly acidic $\mathrm{pH}$, mimicking specific cellular environments, such as endosomes and lysosomes, the aggregation of $\alpha$-syn is greatly accelerated compared with neutral $\mathrm{pH}$, and this effect has been attributed to a strongly $\mathrm{pH}$-dependent autocatalytic process. The molecular mechanism of this process could be nucleation of monomers on fibril surfaces or fibril fragmentation as outlined in Fig. 1. Here, we address the problem of elucidating the $\alpha$-syn aggregation mechanism, with an aim to distinguish between nucleation on fibril surfaces or fibril fragmentation. We have used seeded aggregation kinetics together with a filter trap assay, differential sedimentation analysis, quartz crystal microbalance studies and two-color super-resolution microscopy using dSTORM to distinguish between these two possibilities. Our data imply that the secondary process consists of nucleation of monomers on the surfaces of existing fibrils, hence rationalizing the autocatalytic nature of the aggregation process.

\section{Results}

\subsection{Non-seeded aggregation kinetic experiments}

$\alpha$-Syn aggregation is strongly influenced by intrinsic and extrinsic factors. In order to obtain a well-controlled system, all kinetic studies were here performed under quiescent conditions at mildly acidic $\mathrm{pH}$ (10 mM MES buffer $\mathrm{pH}$ 5.5). To investigate the role of surfaces, reactions starting from monomeric $\alpha$-syn were monitored in untreated polystyrene (PS) plates as well as non-binding PS plates coated with 
polyethylene glycol (PEG). Interestingly, no aggregation of $\alpha$-syn was detected during the time frame of the experiment (up to $100 \mathrm{~h}$ ) in the PEGylated plates, whereas reproducible kinetic traces with typical sigmodal curves were observed in PS plates (S3A). This highlights the importance of heterogeneous nucleation of $\alpha$-syn on the PS surface as previously found when adding PS nanoparticles to $\alpha$-syn in the PEGylated plates (Vácha et al. 2014). Another striking observation from the current experiments in PS plates is that $\alpha$-syn aggregation kinetics appear independent of the peptide concentration at high monomer concentrations (above ca. 30 $\mu \mathrm{M}, \mathrm{S} 3 \mathrm{~B})$. To characterize the aggregation mechanism of $\alpha$-syn in the absence of catalyzing foreign surfaces, all further experiments presented here were conducted in non-binding PEGylated plates in the presence of seeds.

\subsection{Seeded kinetic experiments}

It is well established that by using preformed fibrils as seeds, the primary nucleation reaction can be bypassed (Buell et al. 2014; Giehm \& Otzen, 2010). Therefore, the reaction was here monitored in the presence of controlled amounts of $\alpha$-syn seeds. As the reaction is very sensitive to the concentration and size of the seeds, it is vital to handle all seed solutions in the same manner, as described in the materials and methods section. Initial seeded kinetic experiments were conducted by systematically varying monomer (1-50 $\mu \mathrm{M})$ and seed $(0 \cdot 3-3 \mu \mathrm{M})$ concentrations (Figs 2 and 3 ). The monomer concentrations fall within the physiologically relevant range (Rabe et al. 2013). Strikingly, for each seed concentration, the $\alpha$-syn aggregation rate appears to be independent of the initial monomer concentration above ca. $10 \mu \mathrm{M}$, as evident from the overlapping curves at early times in Fig. $2 \mathrm{a}-\mathrm{c}$. This implies that the processes contributing to the aggregation reaction under these conditions, elongation and secondary nucleation are saturated.

Comparing the kinetic curves at a fixed monomer concentration and varied amount of preformed seeds (Fig. 3), it is clear that increasing concentration of seed fibrils leads to a decrease in lag time, and that no aggregation is observed in the absence of seed fibrils. This behavior is observed at all monomer concentrations studied. The formation of new fibrils is thus strongly enhanced by the presence of seed fibrils, which is the definition of a secondary process (Cohen et al. 2012). In addition, most of the aggregation curves have concave shapes, i.e. an accelerating rate of aggregation at early times, indicating the existence of a process that significantly increases the number of growth competent ends (i.e. aggregates) (S5).

A number of kinetic models were globally fitted to the experimental data (Meisl et al. 2016a) and only models that include secondary processes were found to produce reasonable fits. There are some remaining deviations that may be due to higher-order assembly events (Buell et al. 2014) not included in the existing model. Simulated macroscopic traces for the case of monomer concentration variation in the presence of $1 \mu \mathrm{M}$ seed concentration (experimental data shown in Fig. 2b), for the different kinetic models tested are shown in Fig. 2d-f (Meisl et al. 2016a). Two models were most consistent with the data: having either fragmentation or saturated secondary nucleation as the dominant processes of fibril multiplication (Fig. 2e, f). This is manifested in the very weak dependence of the overall aggregation rate on the monomer concentration observed in our seeded kinetic data. We next designed experiments to distinguish between fragmentation and nucleation of monomers on fibril surface as the dominant secondary process.

\subsection{Sedimentation analysis of fibril size development}

The fragmentation of fibrils would lead to a change in fibril size distribution, even after the monomer concentration has reached the solubility limit (Michaels et al. 2015). $\alpha$-Syn aggregates at different times after the aggregation process was completed were investigated using differential sedimentation analysis (Arosio et al. 2016). The method relies on the fact that aggregates of different sizes travel through a sucrose gradient at different speeds. The results show that the retention time profile of the samples remains unaltered over up to 20 days (Fig. 4a), implying that the fragmentation rate is undetectably low under quiescent conditions.

\subsection{Trap and seed kinetic experiment}

As a further test as to whether fragmentation or surface catalysis are the major sources of new aggregates, a set of experiments, referred to as the trap and seed kinetic approach (Arosio et al. 2014) was performed at $37^{\circ} \mathrm{C}$ under quiescent conditions (Fig. 4b). Mature fibrils were trapped in low-binding GHP membrane filter plates (Nasir et al. 2015) with $200 \mathrm{~nm}$ cutoff (retentate), and the flow through was collected in non-binding PEGylated plates (filtrate 1). Purified $\alpha$-syn monomer at different concentrations or buffer alone was added and incubated with the trapped fibrils for $2 \mathrm{~h}$ and again filtered (filtrates 2 and 3, respectively). The aggregation kinetics of filtrates 1, 2 and 3 were followed by ThT fluorescence (Fig. 4b). For filtrate 1 , no enhanced fluorescence was detected within the time frame of the experiment $(90 \mathrm{~h})$, which indicates that seeds were trapped in the GHP membrane filter, and that any monomer or smaller species in the filtrate are present at too low concentration to give rise to any significant aggregation (Fig. 4B1). This control is relevant to show that the system has reached equilibrium. When $16 \mu \mathrm{M}$ of monomeric $\alpha$-syn was added to wells with filtrate 1 , aggregation was detected. This implies that some catalyzing species are present in filtrate 1 (Fig. 4B1). For filtrate 2, sigmoidal ThT fluorescence curves are seen within ca. $15 \mathrm{~h}$ at all monomer concentrations (Fig. 4B2). In contrast, no fluorescence increase is observed for filtrated monomer that has been 
(a)
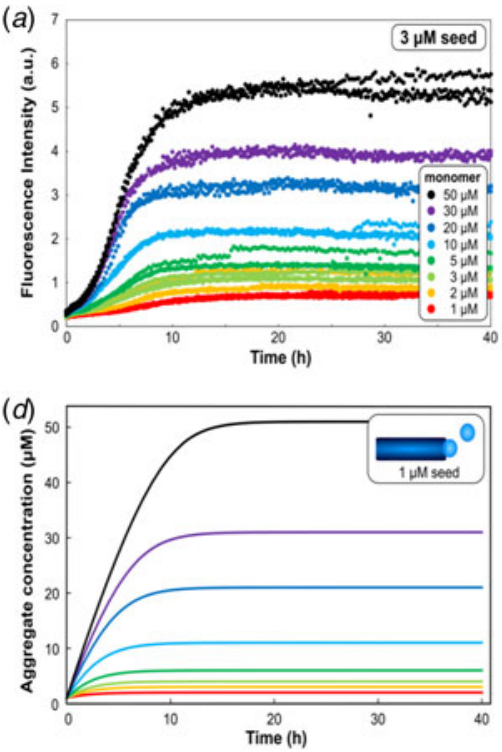

(b)

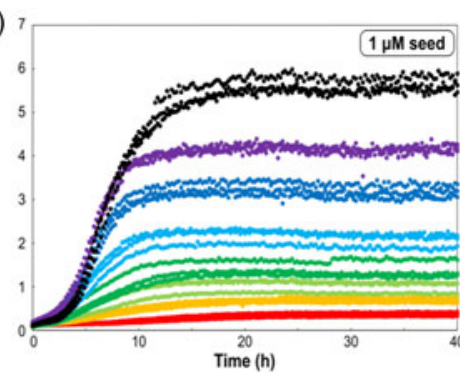

(e)

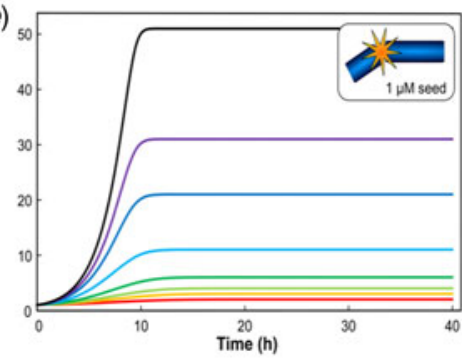

(c)
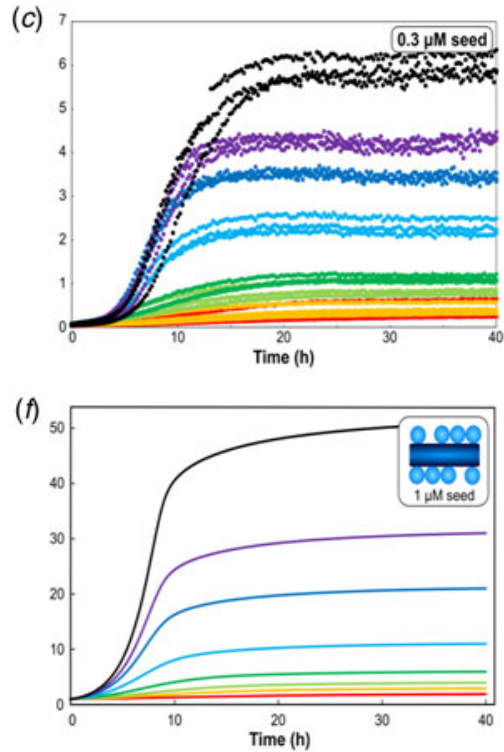

Fig. 2. Seeded $\alpha$-syn aggregation kinetics. The monomer concentration was systematically varied in the range of $1-50 \mu \mathrm{M}$ in the presence of three different seed concentrations: (a) $3 \mu \mathrm{M}$, (b) $1 \mu \mathrm{M}$ and (c) $0.3 \mu \mathrm{M}$ in $10 \mathrm{mM} \mathrm{MES} \mathrm{pH} 5.5$ at $37^{\circ} \mathrm{C}$ under quiescent conditions. For each solution condition three traces are shown in bold circles. The figures show ThT intensity as a function of time (non-normalized raw data). Different microscopic mechanistic events lead to overall different observable macroscopic kinetic profiles, as shown through simulated traces for the case of monomer concentration variation in the presence of $1 \mu \mathrm{M}$ seed (experimental data shown in $2 \mathrm{~b}$ ) for the different kinetic models tested, (d) only primary nucleation and elongation, (e) dominant fragmentation and (f) dominant saturated secondary nucleation.

incubated in the filter plate without seeds (negative control) (Fig. 4B2). Together, this indicates that during incubation of monomer with trapped fibrils, a fibril-catalyzed reaction generates a significant concentration of aggregates that are small enough to pass through the filter together with the remaining monomer. We conclude that the trapped fibrils present catalytic surfaces promoting aggregation of the added monomeric $\alpha$-syn. ThT was added to filtrate 3 and the fluorescence was monitored for approximately $70 \mathrm{~h}$ (Fig. 4B3). The ThT intensity was close to background suggesting either that fibril fragmentation did not occur to any significant extent, or that fragments were larger than the membrane cutoff. After ca. $70 \mathrm{~h}, 16 \mu \mathrm{M}$ monomer was added to filtrate 3 , but no fluorescence increase was detected over the following $80 \mathrm{~h}$, confirming the absence of small catalyzing species that could have been present as a result of fibril fragmentation (Fig. 4B3).

\subsection{Surface affinity of monomers is $\mathrm{pH}$ dependent}

The interaction between monomeric and fibrillar $\alpha$-syn as a function of $\mathrm{pH}$ was studied by means of quartz crystal microbalance with dissipation (QCM-D) to monitor the association and dissociation of monomers to and from surface-attached fibrils. This experimental strategy relies on the observation that fibril elongation occurs at significant rates over a much larger $\mathrm{pH}$ range than secondary nucleation (Buell et al. 2014). The QCM-D measurements provide information on the total amount of adsorbed material (including the coupled solvent) from the change in frequency $(\Delta F)$, as well as the viscoelastic properties of the attached layer from the dissipation $(\Delta D)$. Figure 5 a shows typical QCM-D data where the decrease in frequency is due to added mass, albeit the relation between frequency and mass addition can be non-linear for viscoelastic layers. Gold coated sensor crystals with covalently immobilized and elongated $\alpha$-syn fibrils were exposed to $20 \mu \mathrm{M} \alpha$-syn monomer in $10 \mathrm{mM}$ MES buffer at $\mathrm{pH} 5 \cdot 5,5 \cdot 7,5 \cdot 9,6 \cdot 1$ and $6 \cdot 5$ to allow for monomer adsorption and fibril elongation in different proportions. After a certain frequency shift $(\sim 420$ $\mathrm{Hz}$ ) was obtained for each $\mathrm{pH}$ condition, each sensor was washed with $10 \mathrm{mM}$ MES buffer $\mathrm{pH} 6.5$ to monitor dissociation. We observe a strong $\mathrm{pH}$ dependence of monomer association with the fibrils. A sharp decrease in frequency was observed during incubation with monomer at $\mathrm{pH}<$ $6 \cdot 0$, followed by a rapid increase during washing at $\mathrm{pH} 6.5$ (Fig. 5a). This indicates that the majority of mass that was added at $\mathrm{pH}<6.0$ is reversibly associated. A less sharp decrease in frequency was observed during incubation with monomer at $\mathrm{pH}>6.0$, followed by a smaller increase during washing, indicating that the majority of the mass added is unaffected by washing at $\mathrm{pH} 6.5$ (Fig. 5a). We interpret this behavior as evidence for two types of mass addition, the relative importance of which depends on the $\mathrm{pH}$. At $\mathrm{pH}>6.0$ mostly elongation occurs, and monomers are very slowly dissociating upon washing. At $\mathrm{pH}<6 \cdot 0$, surface binding becomes significant, and the mass addition is reversible upon washing at $\mathrm{pH}>6 \cdot 0$. Therefore, reversible 
(a)

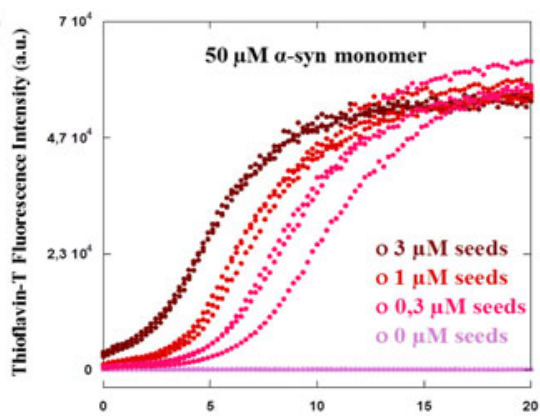

(c)

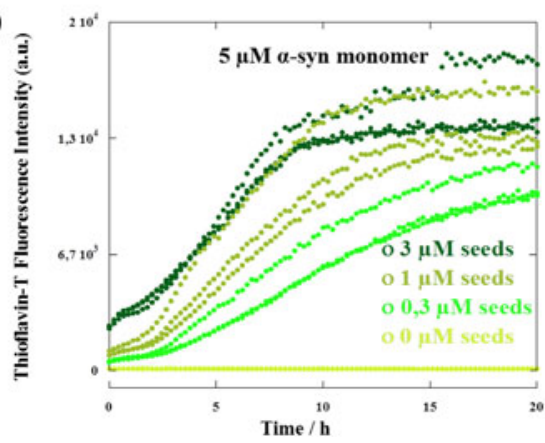

(b)

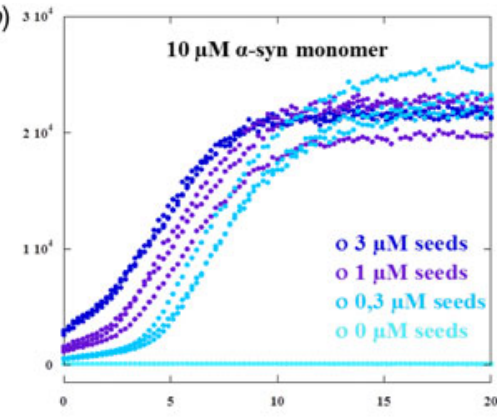

(d)

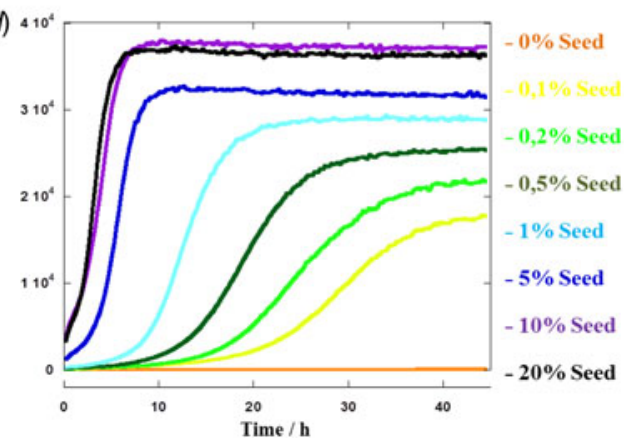

Fig. 3. Seeding efficiency in $\alpha$-syn aggregation. (a-c) Representative seeded aggregation kinetic traces in the presence of fixed ( $3,1,0 \cdot 3$ and $0 \mu \mathrm{M}$ ) seed concentrations incubated with (a) $50 \mu \mathrm{M}$, (b): $10 \mu \mathrm{M}$ and (c) $5 \mu \mathrm{M} \alpha$-syn monomer. For each condition three traces are shown in bold circles. (d) Aggregation with systematic variation of $\alpha$-syn seed concentration from 0 to $20 \%$ in the presence of a fixed $\alpha$-syn monomer concentration $(20 \mu \mathrm{M})$. Averages of three traces are shown as solid lines. All figures show ThT intensity as a function of time (non-normalized raw data). Therefore, experiments in the presence of seeds show elevated ThT intensity at time zero. All experiments were performed in $10 \mathrm{mM}$ MES pH 5.5 in non-binding PEGylated plates at $37^{\circ} \mathrm{C}$ and under quiescent conditions.

surface binding has a similar $\mathrm{pH}$ dependence as the secondary process (Buell et al. 2014), providing additional support for the hypothesis that the secondary process consists of nucleation of monomers on the surface of existing fibrils.

\subsection{Imaging of amyloid growth from seed fibrils}

In order to investigate amyloid growth from seed fibrils at mildly acidic $\mathrm{pH}$ conditions, we imaged samples with superresolution microscopy. The approach employed was a twocolor dSTORM strategy previously reported (Pinotsi et al. 2014). This technique relies on labeling two samples of an $\alpha$-syn cysteine variant (N122C) with two different Alexa Fluor dyes, namely Alexa Fluor 647 (AF647) and Alexa Fluor 568 (AF568). Preformed seeds of $\alpha$-syn-AF647 (purple) were incubated with $\alpha$-syn-AF568 (green) monomer at $\mathrm{pH} 5.5$ (Fig. 6). Comparing with images taken at neutral $\mathrm{pH}$ (Pinotsi et al. 2014), it is again clear that the aggregation mechanism is $\mathrm{pH}$ dependent. Two scenarios were discerned from these images: at mildly acidic $\mathrm{pH}$ monomer showed affinity for the surface along the sides of the existing seeds (co-localization of purple and green in Fig. 6), while for neutral $\mathrm{pH}$ conditions elongation dominated and monomer showed greater affinity for the growth-competent fibril ends (Pinotsi et al. 2014). It should be noted here that, irrespective of the conditions during sample preparation, the dSTORM imaging was performed in a dedicated imaging buffer at $\mathrm{pH} 8 \cdot 2$. As the majority of the mass added at mildly acidic $\mathrm{pH}$ is reversibly associated, as inferred from the QCM-D data (Fig. 5), it is likely that the majority of the mass added to the fibril seeds at $\mathrm{pH} 5.5$ detach from the fibrils when they are placed in buffer of higher $\mathrm{pH}$ due to repulsive electrostatic interactions. This might then lead to an underestimation of the amount of associated $\alpha$-syn-AF568 species at the fibril surface. Still, it is evident that the surfaces along the $\alpha$-syn-AF647 fibrils contain along their length $\alpha$-syn-AF568 species.

\section{Discussion}

Alterations in the balance of protein synthesis and clearance may lead to the formation of toxic oligomers and trigger a neurodegenerative cascade (Lee et al. 2012). No clear correlation has been found between the amount of $\alpha$-syn inclusions and the stage of PD (Chaudhary et al. 2014), although larger areas of the brain contain aggregated $\alpha$-syn as the disease progresses (Braak et al. 2002).

In a previous study, we have shown that the $\alpha$-syn aggregation is dominated by secondary processes under mildly acidic conditions $(\mathrm{pH}<6.0)$ (Buell et al. 2014). It was also shown that at neutral $\mathrm{pH}$, there is a linear relation between the elongation rate and monomer concentration at low monomer concentrations, and that the elongation rate 
(a) Differential Sedimentation
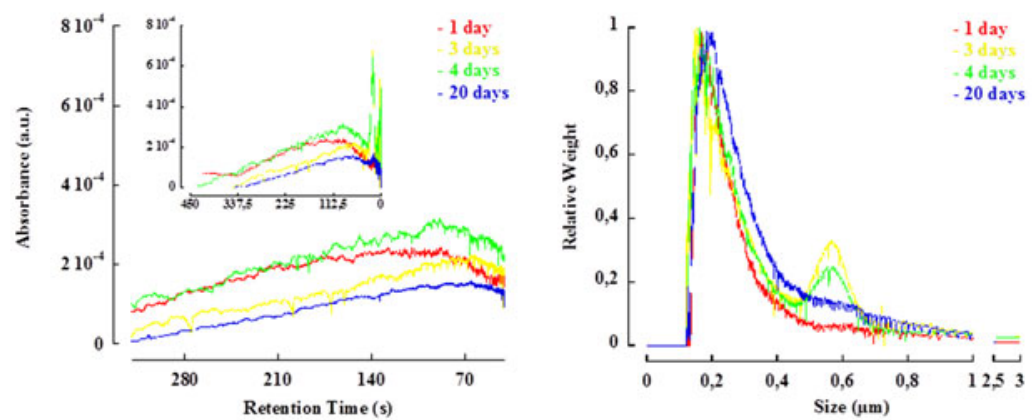

(b) Trap and Seed B1 - Trapping Fibrils

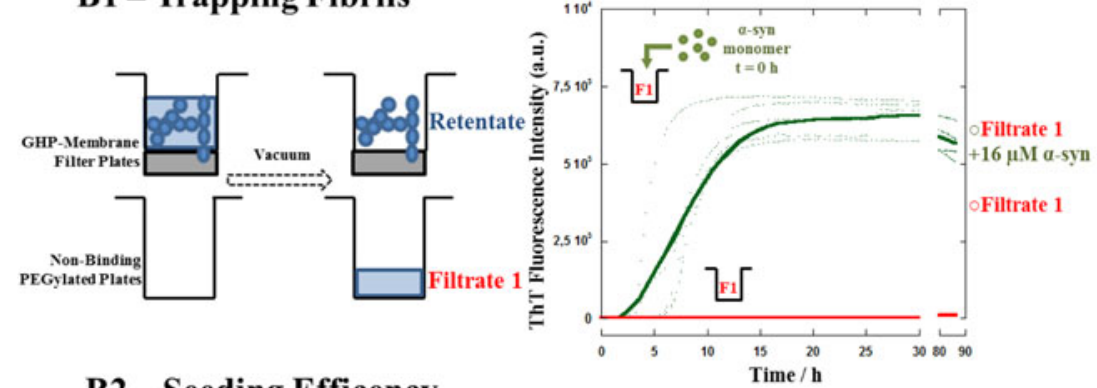

B2 - Seeding Efficency
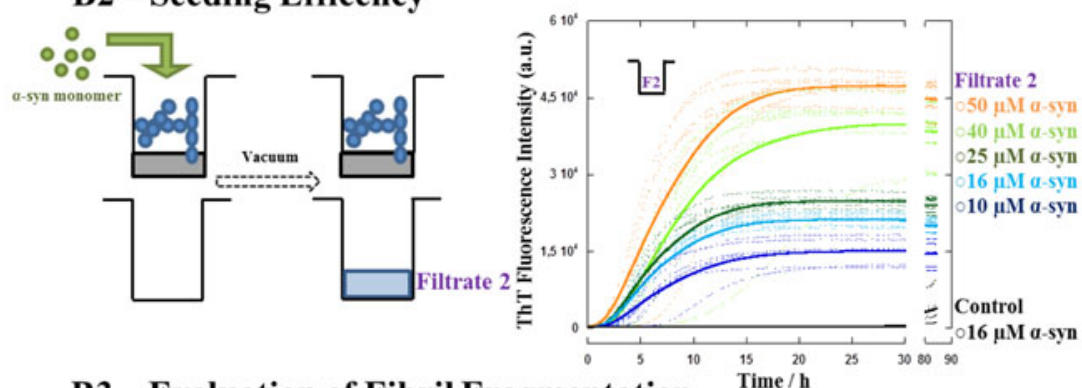

B3 - Evaluation of Fibril Fragmentation
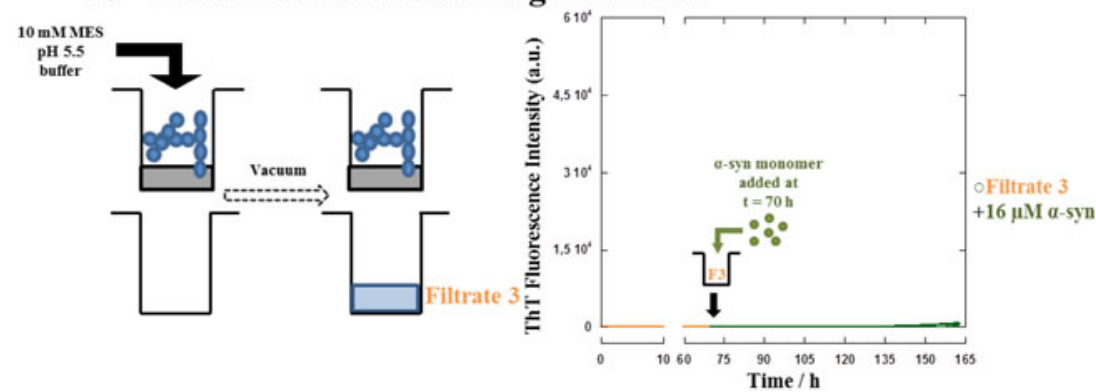

Fig. 4. Experiments designed to identify the dominant secondary process of $\alpha$-syn aggregation at mildly acidic pH conditions. (a) Time-dependent differential sedimentation analysis performed on fibrils incubated at $37^{\circ} \mathrm{C}$ under quiescent conditions in $10 \mathrm{mM}$ MES buffer $\mathrm{pH} 5.5$ for 1-20 days. Aggregates sediment within a sucrose gradient on a rotating disc where longer retention times correspond to smaller size aggregates. The raw data is shown to the left and processed data to the right. The calculations of relative weight and size are made under the assumption of spherical particles, which leads to an underestimation of the size and relative weight for a fibrillar particle. Nevertheless, changes in size distribution can be detected, which was the purpose of this experiment. The figures show representative traces of each condition that was repeated at least two times. (b) Trap and seed kinetic experiment. Fibrils made from $20 \mu \mathrm{M} \alpha$-syn monomer supplemented with $3 \mu \mathrm{M}$ seed fibrils in $10 \mathrm{mM}$ MES pH 5.5 were trapped by filtration in filter plates and the flow-through (filtrate 1) was collected in non-binding PEGylated plates, supplemented with ThT and monitored in a plate reader (B1). The trapped fibrils were then incubated for $2 \mathrm{~h}$ with concentrations ranging from 10 to $50 \mu \mathrm{M} \alpha$-syn monomer or $10 \mathrm{mM}$ MES buffer pH 5.5 and newly filtered (filtrates 2 and 3, respectively). Again, the flow-through was collected in non-binding PEGylated plates, supplemented with ThT and monitored (B2 and B3). The figures show averages of at least four traces that are shown in bold with individual traces dotted below and are plotted as ThT intensity as a function of time (non-normalized data). 

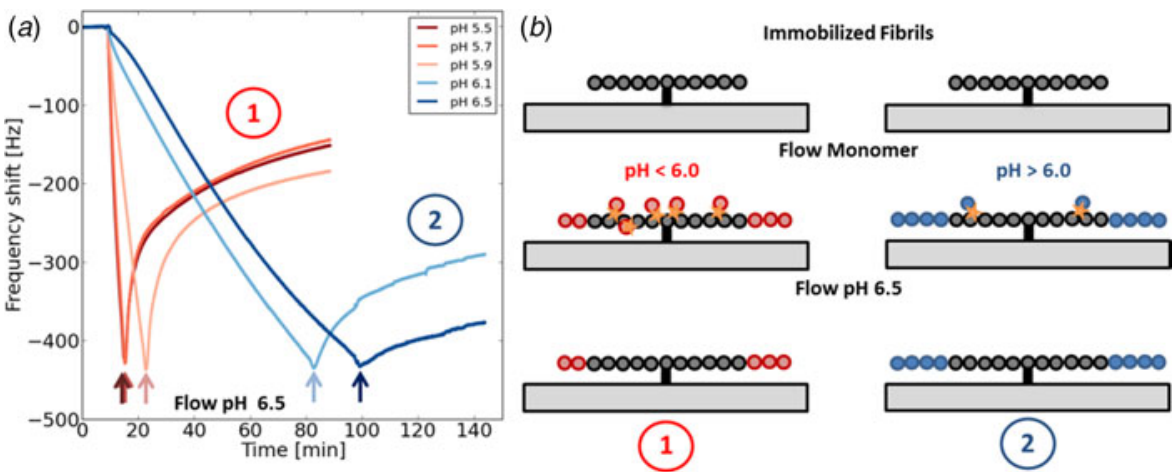

Fig. 5. The surface of $\alpha$-syn amyloid fibrils shows a pH-dependent affinity for monomeric $\alpha$-syn. (a) Fibrils of $\alpha$-syn were immobilized on gold-coated QCM-D sensor crystals and incubated with $20 \mu \mathrm{M}$ monomeric $\alpha$-syn in $10 \mathrm{mM}$ MES buffer at different $\mathrm{pH}$ values until a certain frequency shift was reached (ca. $-420 \mathrm{~Hz}$ for overtone $N=3$ ). The sensors were then washed with $10 \mathrm{mM}$ MES pH 6.5 (indicated by arrows), leading to different levels of dissociation of the previously attached material. (b) Scheme of what is postulated to occur on the sensors for the conditions of $\mathrm{pH}<6.0$ and $\mathrm{pH}>6 \cdot 0$. The orange stars represent binding to the surface of the fibrils.

becomes saturated at high monomer concentrations (Buell et al. 2014). Similar overall behavior has been observed for other amyloid proteins, including Sup35 yeast protein (Collins et al. 2004), S6 (Lorenzen et al. 2012), insulin (Buell et al. 2010a) and $\alpha$-lactalbumin (Buell et al. 2010a).

In the current work, we aim to understand the nature of the dominant secondary process at mildly acidic $\mathrm{pH}$. As a first strategy we studied the monomer dependence of the aggregation kinetics in the presence of low concentrations of preformed seed fibrils. Two different scenarios are consistent with the observation that the aggregation kinetics is only weakly dependent on the concentration of monomeric $\alpha$-syn: (a) fibrils multiply through fragmentation or (b) fibrils catalyze the formation of new aggregates from monomers on their surface, but this reaction is saturated at the monomer concentrations investigated. Secondary nucleation consists of an initial attachment of monomers to the surface, followed by nucleus formation and detachment. However, secondary nucleation may saturate at high enough monomer concentrations, where the initial attachment becomes very fast. The fibril is then fully covered in monomer and the second, monomer-independent (rearrangement or detachment) step, becomes rate limiting (Meisl et al. 2014). The two models lead to the same overall scaling of the kinetic behavior with monomer and fibril concentration. To distinguish the nature of the dominant secondary mechanism, additional and complementary experiments were performed including: (i) differential sedimentation, (ii) the trap and seed kinetic approach, (iii) QCM-D biosensing studies and (iv) two-color super-resolution microscopy using dSTORM.

Fragmentation and secondary nucleation mechanisms may lead to different final states. If fragmentation is the dominant mechanism, then the mature fibrils will keep fragmenting even after completion of the aggregation reaction. However, the differential sedimentation analysis shows that the mature fibrils do not become shorter over time, implying that spontaneous fragmentation occurs very slowly. This makes fragmentation of fibrils less likely to be the dominant secondary mechanism.

The trap and seed kinetic approach was used to probe whether new oligomeric aggregates smaller than $200 \mathrm{~nm}$ are formed during a reaction involving both monomer and fibrils. We found that $\alpha$-syn aggregation is clearly faster in samples of filtrates from reactions where $\alpha$-syn monomers had been incubated with trapped fibrils compared with samples where $\alpha$-syn monomers had been incubated for the same time in the same type of filters with no preformed seeds, confirming again the importance of secondary processes. Moreover, since shedding/fragmentation of the trapped fibrils when incubated with buffer alone under the same experimental conditions was undetectable, alongside the observation that spontaneous fragmentation does not occur during the time frame studied, we propose that the newly formed oligomeric species are most likely generated by a secondary nucleation process of $\alpha$-syn monomer at the surface of existing fibrils. As with the seeded kinetic experiments, in the trap and seed assay, we also observed that the aggregation kinetics are to a large extent independent of the monomer concentration, likely a result of saturated secondary nucleation.

QCM-D experiments were set up with changes in $\mathrm{pH}$ between the association and dissociation phases to probe whether the added aggregate mass would be reduced at different rates upon washing with buffer at $\mathrm{pH} 6.5$, depending on the $\mathrm{pH}$ value during mass association. The QCM-D results reveal that part of the mass associated with the surface-bound fibrils at low $\mathrm{pH}$, dissociates upon $\mathrm{pH}$ increase, which is likely due to release of protein that is relatively weakly attached to the surface of the fibrils, i.e. monomeric protein or indeed already formed secondary nuclei. On the other hand, at $\mathrm{pH}>6.0$ mostly elongation occurs, and monomers dissociate very slowly upon washing due to the high thermodynamic stability 

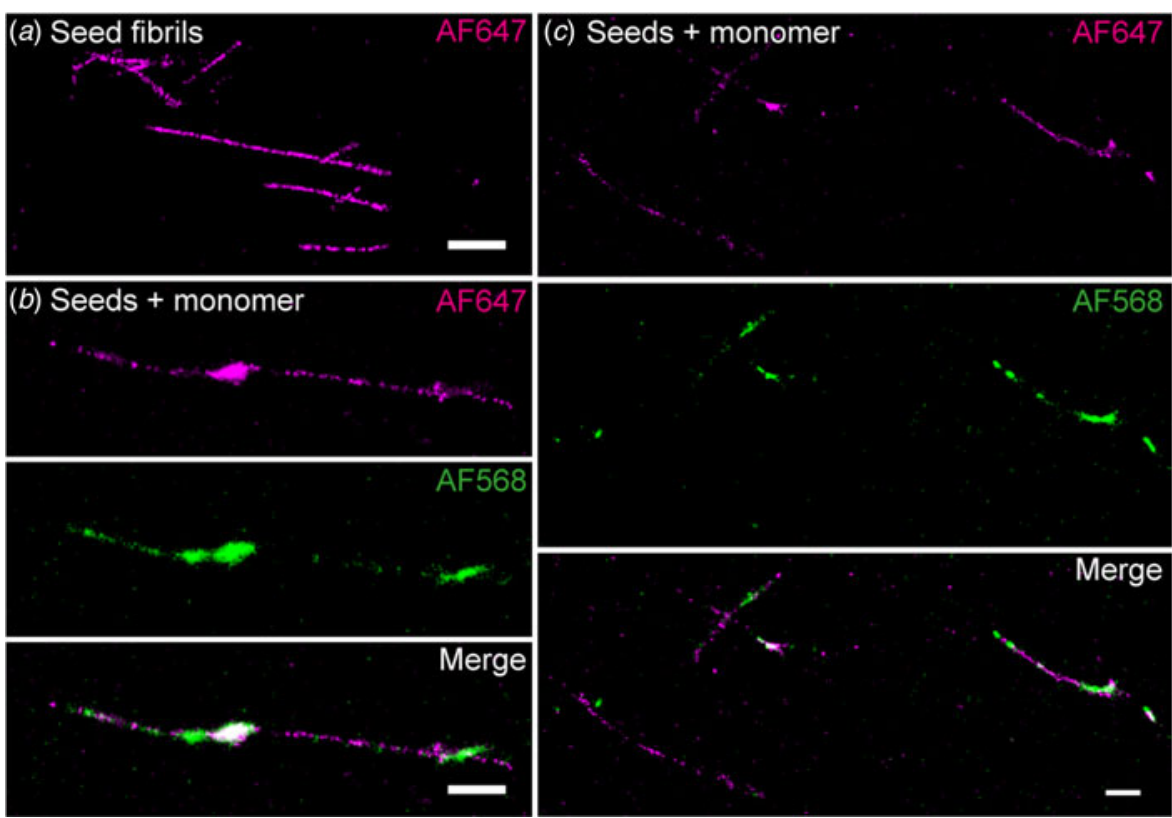

Fig. 6. $d$ STORM images of amyloid growth from seed fibrils at mildly acidic $\mathrm{pH}$ conditions. (a) Imaged preformed seed fibrils of $\alpha$-syn N122C labeled with 1:20 AF647 used for the self-seeding experiment. (b, c) $\alpha$-Syn monomer labeled with AF568 (green) was incubated with 50\% seed fibrils labeled with AF647 (purple). The top panel is imaged in the red channel, middle panel imaged in the green channel and the bottom panel is the merge between both channels. Scale bar corresponds to $1 \mu \mathrm{m}$.

of the fibrils (Baldwin et al. 2011). The QCM-D experiments, also support that nucleation of new fibrils from surface-bound monomers is the most likely secondary process at mildly acidic $\mathrm{pH}$. The dependence on $\mathrm{pH}$ of $\alpha$-syn monomer surface affinity was also evident in the two-color dSTORM images. Here, it was clearly shown that at mildly acidic $\mathrm{pH}$ conditions, a larger proportion of monomer is bound at the surface along the seed fibrils, opposing to neutral conditions, where monomer has higher affinity for the growth-competent fibril-free ends (Pinotsi et al. 2014).

In summary, the combination of experiments presented here very strongly suggests that the nucleation of monomers on fibril surfaces is the secondary process that dominates the aggregation mechanism for $\alpha$-syn at $\mathrm{pH}<6 \cdot 0$. Thus, at mildly acidic $\mathrm{pH}$ the aggregation of $\alpha$-syn follows the same mechanism as previously found for insulin (Foderà et al. 2008), IAPP (Padrick \& Miranker, 2002) and the amyloid $\beta$ peptide, $\mathrm{A} \beta$ (Cohen et al. 2013; Meisl et al. 2014). Although surface catalyzed secondary nucleation dominates the aggregation process for $\mathrm{A} \beta$ also at basic $\mathrm{pH}$, a reduction in $\mathrm{pH}$ lowers the electrostatic repulsion between monomers and fibrils leading to enhanced secondary nucleation (Meisl et al. 2016b). $\alpha$-Syn has a pI of 4.7 (Uversky et al. 2001) and carries a net negative charge at neutral $\mathrm{pH}$. The sequence is separated into three distinct regions, with a large number of charged residues in the $\mathrm{N}$ - and $\mathrm{C}$-terminal regions and a higher fraction of hydrophobic residues in the central region. Interactions involving hydrophobic groups may play a significant role at all $\mathrm{pH}$ values, but at neutral $\mathrm{pH}$, there is significant electrostatic repulsion between monomers, as well as, between monomers and fibrils. The charge of the residues in the termini is modulated by $\mathrm{pH}$ and therefore the electrostatic interactions between monomers and fibrils are likely to have strong $\mathrm{pH}$ dependence. This may underlie the observed $\mathrm{pH}$ dependence of secondary nucleation (Buell et al. 2014).

It can be postulated that changes in $\mathrm{pH}$ between different cellular environments may affect the rate of formation of potentially cytotoxic oligomeric species, as observed under the conditions studied here. Mildly acidic $\mathrm{pH}$ is found in many intracellular compartments, linked to the endocytic and exocytic pathways, making this set of conditions physiologically relevant. In vitro studies at mildly acidic $\mathrm{pH}$ can thus bring new insights into the microscopic steps underlying the aggregation mechanism of $\alpha$-syn, and in particular, aggregate multiplication in the brain (Buell et al. 2014). The secondary nucleation process of $\alpha$-syn monomers on fibril surfaces described here is an autocatalytic process, which constitutes a double threat; it increases rapidly both the total load of fibril mass and the amount of smaller oligomeric species that are thought to be the main responsible agents for cytotoxicity (Xue et al. 2009). Since secondary nucleation appears as the dominant route to $\alpha$-syn aggregation at mildly acidic $\mathrm{pH}$, this reaction can be considered a future therapeutic target for development of small molecular inhibitors or using certain chaperones that have been successfully shown to inhibit this specific microscopic process for the $\mathrm{A} \beta$ peptide (Cohen et al. 2015). 


\section{Material and method section}

\subsection{Recombinant $\alpha$-syn peptide expression and purification}

Human $\alpha$-syn WT and $\alpha$-syn cysteine variant (N122C) were expressed and purified using heat treatment, ion exchange and gel filtration chromatography as described previously (Grey et al. 2011) (S1). Labeling of $\alpha$-syn cysteine variant (N122C) with Alexa Fluor 647 and Alexa Fluor 568 was performed as previously reported (Pinotsi et al. 2014) (S6).

\subsection{ThT kinetic experiments}

A key factor in achieving reproducible kinetics is to use pure monomeric $\alpha$-syn as starting material. Prior to setting up any kinetic measurement, the frozen aliquots were purified with a final size exclusion chromatography run in $10 \mathrm{mM}$ MES pH 5.5 (standard condition) were the central region of the peak is collected in order to assure the presence of only monomeric species (S1). The protein concentration was determined by measuring the UV absorbance at 280 $\mathrm{nm}$ and using the extinction coefficient $\varepsilon_{280}=5800 \mathrm{lmol}^{-1}$ $\mathrm{cm}^{-1}$. Buffer solutions were filtered and degassed before each run. The isolated monomeric $\alpha$-syn was always kept on ice to prevent aggregation.

To follow the fibrillation process, samples were aliquoted in 96-well plates with a non-binding surface (black PS plates treated with a PEGylated surface, half-area, 3881 Corning), supplemented with $20 \mu \mathrm{M}$ of ThT and sealed with a plastic film to avoid evaporation. With the exception being for the experiment to investigate the role of surfaces on $\alpha$-syn aggregation shown in S3, were also non-treated surface (black PS plates, full volumes, 3631 Costar) were used. The optimal concentration for ThT $(20 \mu \mathrm{M})$ was accessed to have a linear relationship between fluorescence intensity and aggregate concentration (S2). Plates were incubated at $37^{\circ} \mathrm{C}$ up to $100 \mathrm{~h}$ in a plate reader (FluoStar Omega or FluoStar Galaxy, BMG Labtech, Offenburg, Germany) under quiescent conditions (excitation filter $440 \mathrm{~nm}$ and emission filter $480 \mathrm{~nm}$ ).

\subsection{Preparation of $\alpha$-syn seed fibrils}

$\alpha$-Syn seed fibrils were formed in Eppendorf tubes (Axygen low-bind tubes) at $37^{\circ} \mathrm{C}$, with low stirring speed (300 rpm) with a teflon bar and left fibrillating for up to $48 \mathrm{~h}$. Parallel kinetic ThT measurements were performed to assure that the plateau was reached during this time period. Before each kinetic experiment, seed fibrils were pre-treated by $1 \mathrm{~min}$ continuous sonication at maximum power in a sonicator bath (Struer, Copenhagen, DK) to disperse lumped fibrils. The concentration of seeds is counted as monomer equivalents.

\subsection{Differential sedimentation method}

In order to measure retentions times of the main populations of mature fibrils as a function of time a sedimentation analysis was performed using a CPS disc centrifuge instrument model DC24000. The instrument was operated at $13782 \mathrm{rpm}$ and a 4 to $12 \%$ sucrose gradient was cast in the spin fluid (Milli-Q water). Dodecane was added last to the gradient to extend its lifetime. Calibration was done with polyvinyl chloride particles with a weight-average diameter of $483 \mathrm{~nm}$, and $100 \mu \mathrm{l}$ samples were injected.

\subsection{Trap and seed}

Fibrils were made from $20 \mu \mathrm{M}$ monomeric $\alpha$-syn in the presence of $3 \mu \mathrm{M}$ seeds under quiescent conditions at $37^{\circ} \mathrm{C}$ in non-binding PEGylated plates. The fibrils were trapped on the filter membranes (retentate) by filtration applying vacuum for $10 \mathrm{~s}$ on a low-binding AcroPrep 96-well filter plate (plate housing - Polypropylene) embedded with a Versatile GH Polypro membrane (GHP - hydrophilic Polypropylene membrane) (Pall Life Sciences, Ann Arbor, MI). The flow through was collected in a 96-well nonbinding PEGylated plate (filtrates). The filtration was done using a MultiScreenHTS vacuum (Millipore) manifold. Before loading the seed samples, the GHP filter membrane of the multi-well plate was washed with experimental buffer. The fluorescence intensity of ThT of each filtrate collected in the non-binding PEGylated plates was monitored in a plate reader at $37^{\circ} \mathrm{C}$ under quiescent conditions.

\subsection{QCM-D measurements}

The QCM-D measurements were performed with an E4 instrument (Q-Sense, Västra Frölunda, Sweden). $\alpha$-Syn fibrils were produced and attached to the gold-coated surface of a quartz sensor (QSX 301) as previously described (Buell et al. 2010b, 2012) (S4). For the main experiments, the sensors were incubated with solutions of $20 \mu \mathrm{M}$ monomeric $\alpha$-syn in $10 \mathrm{mM}$ MES buffer at the $\mathrm{pH}$ values of 5.5, 5.7, 5.9, $6 \cdot 1$ and $6 \cdot 5$. When the frequency shift had reached approximately $420 \mathrm{~Hz}$ in the frequency overtone $N=3$, the sensor surface was washed with $10 \mathrm{mM}$ MES buffer at $\mathrm{pH} 6 \cdot 5$.

\subsection{Two-color super-resolution microscopy using dSTORM}

Two-color super-resolution microscopy was performed on an inverted total internal reflectance fluorescence microscope which was custom-built for dSTORM imaging, as described previously (Kaminski Schierle et al. 2011; Pinotsi et al. 2014). Alexa Fluor 647 and 568 dyes were excited using a $640 \mathrm{~nm}$ diode laser (Toptica) and a 561 $\mathrm{nm}$ DPSS laser (Oxxius), respectively with an irradiance between 1 and $5 \mathrm{~kW} \mathrm{~cm}^{-2}$, and a $405 \mathrm{~nm}$ laser diode was used as a reactivation source. The fluorescence was collected with a 100X/1.49 NA objective (Nikon) onto an EMCCD camera (iXon3 897, Andor). To induce photoswitching of the dye the fibrils were immersed in a switching buffer consisting of $100 \mathrm{mM}$ mercaptoethylamine (Sigma) in PBS at $\mathrm{pH} 8.2$ supplemented with an oxygen 
scavenger to reduce photobleaching $\left(40 \mathrm{mg} \mathrm{ml}^{-1}\right.$ glucose, 50 $\mu \mathrm{g} \mathrm{ml}^{-1}$ glucose oxidase, $1 \mu \mathrm{g} \mathrm{ml}^{-1}$ catalase). The red and green channels were imaged sequentially, and for each field of view, a series of 10-15000 frames was acquired with 15 ms exposure time. The acquired dSTORM datasets were analyzed using rapidSTORM 3.3 (Wolter et al. 2012) and superresolution images were generated using a pixel size of $30 \mathrm{~nm}$ pixel $^{-1}$ for both channels. Seeds with different labeling densities were tested for image optimization. Also, these dye labels were evaluated in terms of interfering with the aggregation process using ThT kinetics studies. It was shown that a 1:20 ratio of labeled $\alpha$-syn to unlabeled peptide was the optimal in terms of labeling density and unperturbed aggregation kinetics (S7).

\section{Supplementary Material}

To view supplementary material for this article, please visit https://doi.org/10.1017/S0033583516000172

\section{Acknowledgements}

This work was supported by the Swedish Research Council and its Linneaus Centers for Organizing Molecular Matter (E. Sparr and S. Linse), the European Research Council (S. Linse), Nanolund (S. Linse), Multipark (S. Linse and R. Gaspar), the Leverhulme Trust (A. Buell), Magdalene College, Cambridge (A. Buell), the Parkinson's and Movement Disorder Foundation (A. Buell), EPSRC (C.F. Kaminski), MRC (C.F. Kaminski) and Wellcome Trust UK (C.F. Kaminski). We thank Gunnel Karlsson for the expert help with Cryo-TEM, C. Galvagnion and C.M. Dobson for kindly providing the plasmid for $\alpha$-syn cysteine variant (N122C) expression, G. Schierle Kaminski for helpful inputs and discussions concerning the dSTORM experiments and we would also like to acknowledge K. Sanagavarapu, X. Yang, M. Tornqvist, N. Yu and W.Y. Chen for the support given during the dStorm imaging. We also acknowledge T. Cedervall and S. Gunnarsson for expert help with the fibril sedimentation analysis.

\section{Author Contributions}

R.G., A.K.B, C.K., T.P.J.K., E.S. and S.L. designed the experiments. R.G., A.K.B. and L.Y. performed the experiments. R. G., A.K.B., G.M., L.Y., T.P.J.K, E.S. and S.L. analyzed data. R. G., E.S. and S.L. wrote the paper. All authors contributed to the manuscript writing and revision.

\section{References}

Arosio, P., Cedervall, T., Knowles, T.P. J. \& Linse, S. (2016). Analysis of the length distribution of amyloid fibrils by centrifugal sedimentation. Analytical Biochemistry 504, 7-13.
Arosio, P., Cukalevski, R., Frohm, B., Knowles, T. P. J. \& Linse, S. (2014). Quantification of the concentration of $A \beta 42$ propagons during the lag phase by an amyloid chain reaction assay. Journal of the American Chemical Society 136, 219-225.

Arosio, P., Knowles, T. P. J. \& Linse, S. (2015). On the lag phase in amyloid fibril formation. Physical Chemistry Chemical Physics 17, 7606-7618.

Ashmad, B., Chen, Y. \& Lapidus, L. J. (2012). Aggregation of $\alpha$-synuclein is kinetically controlled by intramolecular diffusion. Proceedings of the National Academy of Sciences of the United States of America 109, 2336-2341.

Baldwin, A. J., Knowles, T. P. J., Tartaglia, G. G., Fitzpatrick, A. W., Devlin, G. L., Shammas, S. L., Waudby, C. A., Mossuto, M. F., Meehan, S., Gras, S. L., Christodoulou, J., Anthony-Cahill, S. J., Barker, P. D., Vendruscolo, M. \& Dobson, C. M. (2011). Metastability of native proteins and the phenomenon of amyloid formation. Journal of the American Chemical Society 133, 14160-14163.

Bisaglia, M., Mammi, S. \& Bubacco, L. (2009). Structural insights on physiological functions and pathological effects of $\alpha$-synuclein. FASEB Journal Review 23, 329-340.

Braak, H., Tredici, K. D., Bratzke, H., Hamm-Clement, J., SAndmann-Keil, D. \& RÜB, U. (2002). Staging of the intracerebral inclusion body pathology associated with idiopathic Parkinson's disease (preclinical and clinical stages). Journal of Neurology 249 (Suppl. 3), III/1-III/5.

Buell, A. K., Blundell, J. R., Dobson, C. M., Welland, M. E., Terentjev, E. M. \& Knowles, T. P. J. (2010a). Frequency factors in a landscape model of filamentous protein aggregation. Physical Review Letters 104, 228101.

Buell, A. K., Dobson, C. M. \& Welland, M.E. (2012). Measuring the kinetics of amyloid fibril elongation using quartz crystal microbalances. Methods in Molecular Biology 849, 101-119.

Buell, A. K., Galvagnion, C., Gaspar, R., Sparr, E., Vendruscolo, M., Knowles, T. P. J., Linse, S. \& Dobson, C. M. (2014). Solution conditions determine the relative importance of nucleation and growth processes in $\alpha$-synuclein aggregation. Proceedings of the National Academy of Sciences of the United States of America 111, 7671-7676. Buell, A. K., White, D. A., Meier, C., Welland, M. E., Knowles, T. P. J. \& Dobson, C. M. (2010b). Surface attachment of protein fibrils via covalent modification strategies. Journal of Physical Chemistry B 114, 10925-10938.

Campioni, S., Carret, G., Jordens, S., Nicoud, L., Mezzenga, R. \& RIEK, R. (2014). The presence of an air-water interface affects formation and elongation of $\alpha$-synuclein fibrils. Journal of the American Chemical Society 136, 2866-2875.

Chaudhary, H., Stefanovi, A., Subramaniam, V. \& Claessens, M. (2014). Membrane interactions and fibrillization of $\alpha$-synuclein play an essential role in membrane disruption. FEBS Letters 588, 4457-4463.

Cohen, S. I. A., Arosio, P., Presto, J., Kurudenkandy, F. R., Biverstål, H., Dolfe, L., Dunning, C. J., Yang, X., Frohm, B., Vendruscolo, M., Johansson, J., Dobson, C. M., Fisahn, A., Knowles, T. P. J. \& LiNSE, S. (2015). A molecular chaperone breaks the catalytic cycle that generates toxic $\mathrm{A} \beta$ oligomers. Nature Structural and Molecular Biology 22, 207-213.

Cohen, S. I. A., Linse, S., Luheshi, L. M., Hellstrand, E., White, D. A., Rajan, L., Otzen, D. E., Vendruscolo, M., Dobson, C. M. \& KNOWLES, T. P. J. (2013). Proliferation of amyloid- $\beta 42$ aggregates occurs through a secondary nucleation mechanism. Proceedings 
of the National Academy of Sciences of the United States of America 110, 9758-9763.

Cohen, S. I. A., Vendruscolo, M., Dobson, C. M. \& Knowles, T. P. J. (2012). From macroscopic measurements to microscopic mechanisms of protein aggregation. Journal of Molecular Biology 421, $160-171$.

Collins, S. R., Douglass, A., Vale, R. D. \& Weissman, J.S. (2004). Mechanism of prion propagation: amyloid growth occurs by monomer addition. PLoS Biology 2, e321.

Conway, K. A., Harper, J. D. \& Lansbury, P. T. (1998). Accelerated in vitro fibril formation by a mutant $\alpha$-synuclein linked to early-onset Parkinson's disease. Natural Medicines 4, 1318-1320.

Danzer, K. M., Krebs, S. K., Wolff, M., Birk, G. \& Hengerer, B. (2009). Seeding induced by $\alpha$-synuclein oligomers provides evidence for spreading of $\alpha$-synuclein pathology. Journal of Neurochemistry 111, 192-203.

Dobson, C. M. (2003). Protein folding and misfolding. Nature 426, 884-890.

FINK, A. L. (2006). The aggregation and fibrillation of $\alpha$-synuclein. Accounts of Chemical Research 39, 628-634.

FoderA, V., Librizzi, F., Groenning, M., van de Weert, M. \& Leone, M. (2008). Secondary nucleation and accessible surface in insulin amyloid formation. Journal of Physical Chemistry B 112, 3853-3858.

Galvagnion, C., Buell, A. K., Meisl, G., Michaels, T. C. T., Vendruscolo, M., Knowles, T. P. J. \& Dobson, C. M. (2015). Lipid vesicles trigger $\alpha$-synuclein aggregation by stimulating primary nucleation. Nature Chemical Biology 11, 229-234.

Gienm, L. \& OtZen, D. E. (2010). Strategies to increase the reproducibility of $\alpha$-synuclein in plate reader assays. Analytical Biochemistry 400, 270-281.

Grey, M., Dunning, C. J., Gaspar, R., Grey, C., Brundin, P., Sparr, E. \& LINSE, S. (2015). Acceleration of $\alpha$-synuclein aggregation by exosomes. Journal of Biological Chemistry 290, 2969-2982.

Grey, M., Linse, S., Nilsson, H., Brundin, P. \& Sparr, E. (2011). Membrane interaction of $\alpha$-synuclein in different aggregation states. Journal of Parkinson's Disease I, 359-371.

izawa, Y., Tateno, H., Kameda, H., HiraKawa, K., Hato, K., Yagi, H., Hongo, K., Mizobata, T. \& Kawata, Y. (2012). Role of $\mathrm{C}$-terminal negative charges and tyrosine residues in fibril formation of $\alpha$-synuclein. Brain and Behaviour 2, 595-605.

Jan, A., Adolfsson, O., Allaman, I., Buccarello, A., Magistretti, P. J., Pfeifer, A., Muhs, A. \& Lashuel, H. A. (2011). A $\beta 42$ neurotoxicity is mediated by ongoing nucleated polymerization process rather than by discrete $\mathrm{A} \beta 42$ species. Journal of Biological Chemistry 286, 8585-8596.

Kaminski Schierle, G. S., van de Lind, S., Erdely, M., Esbjörner, E. K., Klein, T., Rees, E., Bertoncini, C. W., Dobson, C. M., Sauer, M. \& Kaminski, C. F. (2011). In situ measurements of the formation and morphology of intracellular $\beta$-amyloid fibrils by superresolution fluorescence imaging. Journal of the American Chemical Society 133, 12902-12905.

Lee, S., Desplats, P., Lee, H., Spencer, B. \& Masliah, E. (2012). Cell-to-cell transmission of $\alpha$-synuclein aggregates. Amyloid Proteins: Methods and Protocols, Methods in Molecular Biology 849, 347-361.

Lesné, S., KoH, M. T., Kotilinek, L., Kayed, R., Glabe, C. G., Yang, A., Gallagher, M. \& Ashe, K. H. (2006). A specific amyloid- $\beta$ protein assembly in the brain impairs memory. Nature 440, 352-357.

Lorenzen, N., Cohen, S. I. A., Nielsen, S. B., Herling, T. W., Christiansen, G., Dobson, C. M., Knowles, T. P. J. \& Otzen, D.
(2012). Role of elongation and secondary pathways in S6 amyloid fibril growth. Biophysical Journal 102, 2167-2175.

Meisl, G., Kirkegaard, J. B., Arosio, P., Michaels, T. C., Vendruscolo, M., Dobson, C. M., Linse, S. \& Knowles, T. P. J. (2016a). Molecular mechanisms of protein aggregation from global fitting of kinetic models. Nature Protocols 11, 252-272.

Meisl, G., Yang, X., Frohm, B., Knowles, T. P. J. \& Linse, S. (2016b). Quantitative analysis of intrinsic and extrinsic factors in the aggregation mechanism of Alzheimer-associated $\mathrm{A} \beta$-peptide. Scientific Reports 6, 18728.

Meisl, G., Yang, X., Hellstrand, E., Frohm, B., Kirkegaard, J. B. Cohen, S. I. A., Dobson, C. M., Linse, S. \& Knowles, T. P. J. (2014). Differences in nucleation behavior underlie the contrasting aggregating kinetics of the $\mathrm{A} \beta 40$ and $\mathrm{A} \beta 42$ peptides. Proceedings of the National Academy of Sciences of the United States of America 111, 9384-9389.

Michaels, T. C. T., Yde, P., Willis, J. C. W., Jensen, M. H., Otzen, D., Dobson, C. M., Buell, A. K. \& Knowles, T. P. J. (2015). The length distribution of frangible biofilaments. The Journal of Chemical Physics 143, 164901.

Munishina, L. A., Henriques, J., Uversky, V. N. \& Fink, A. L. (2004). Role of protein-water interactions and electrostatics in $\alpha$-synuclein fibril formation. Biochemistry 43, 3289-3300.

NASIR, I., Linse, S. \& CABALEIro-LAGo, C. (2015). Flourescent filter-trap assay for amyloid fibril formation kinetics in complex solutions. ACS Chemical Neuroscience 6, 1436-1444.

Oosawa, F. \& Asakura, S. (1975). Thermodynamics of the Polymerization of Protein. Waltham, MA: Academic Press.

Padrick, S. B. \& MirankeR, A. D. (2002). Islet amyloid: phase partitioning and secondary nucleation are central to the mechanism of fibrillogenesis. Biochemistry 41, 4694-4703.

Pinotsi, D., Buell, A. K., Galvagnion, C., Dobson, C. M., Schierle, G. S. K. \& KAMINSKI, C. F. (2014). Direct observation of heterogeneous amyloid fibril growth kinetics via two-color superresolution microscopy. Nano Letters 14, 339-345.

Pinotsi, D., Michel, C. H., Buell, A. K., Laine, R. F., Mahou, P., Dobson, C. M., Kaminski, C. F. \& Schierle, G.S. K. (2016). Nanoscopic insights into seeding mechanisms and toxicity of $\alpha$-synuclein species in neurons. Proceedings of the National Academy of Sciences of the United States of America 113, 38153819.

Rabe, M., Soragni, A., Reynolds, N. P., Verdes, D., Liverani, E., Riek, R. \& SEEgER, S. (2013). On-surface aggregation of $\alpha$-synuclein at nanomolar concentrations results in two distinct growth mechanisms. ACS Chemical Neuroscience 4, 408-417.

Schulz-SChaffFer, W. J. (2010). The synaptic pathology of $\alpha$-synuclein aggregation in dementia with Lewy bodies, Parkinson's disease and Parkinson's disease dementia. Acta Neuropathologica 120, 131-143.

Shankar, G. M., Li, S., Mehta, T. H., Garcia-Munoz, A., Shepardson, N. E., Smith, I., Brett, F. M., Farrell, M. A., Rowan, M. J., Lemere, C. A., Regan, C. M., Walsh, D. M., Sabatini, B. L. \& Selkoe, D. J. (2008). Amyloid- $\beta$ protein dimers isolated directly from Alzheimer's brains impair synaptic plasticity and memory. Natural Medicines 14, 837-842.

Stefanis, L. (2012). $\alpha$-Synuclein in Parkinson's disease. Cold Spring Harbor Perspectives in Medicine 4, a009399.

Tanaka, M., Collins, S. R., Toyama, B. H. \& Weissman, J. S. (2006). The physical basis of how prion conformations determine strain phenotypes. Nature 442, 585-589. 
UVERSKY, V. N., LI, J. \& FINK, A. L. (2001). Evidence for a partially folded intermediate in $\alpha$-synuclein formation. Journal of Biological Chemistry 276, 10737-10744.

VÁchA, R., Linse, S. \& Lund, M. (2014). Surface effects on aggregation kinetics of amyloidogenic peptides. Journal of the American Chemical Society 136, 11776-11782.

Wakabayashi, K., Tanji, K., Mori, F. \& Takahashi, H. (2007). The Lewy body in Parkinson's disease: molecules implicated in the formation and degradation of $\alpha$-synuclein aggregates. Neuropathology 27, 494-506.

Winner, B., Jappelli, R., Maji, S. K., Desplats, P. A., Boyer, L., Aigner, S., Hetzer, C., Loher, T., Vilar, M., Campioni, S.,
Tzitzilonis, C., Soragni, A., Jessberger, S., Mira, H., Consiglio, A., Pham, E., Masliah, E., Gage, F. H. \& Riek, R. (2011). In vivo demonstration that $\alpha$-synuclein oligomers are toxic. Proceedings of the National Academy of Sciences of the United States of America 108, 4194-4199.

Wolter, S., Löschberger, A., Holm, T., Aufmkolk, S., Dabauvalle, M., VAN DE LINDE, S. \& SAUER, M. (2012). rapidSTORM: accurate, fast open-source software for localization microscopy. Nature Methods 9, 1040-1041.

Xue, W., Hellewell, A. L., Gosal, W. S., Homans, S. W., Hewitt, E. W. \& RADFord, S.E. (2009). Fibril fragmentation enhances amyloid cytotoxicity. Journal of Biological Chemistry 284, 34272-34282. 